\title{
Grapevine and Wine Metabolomics-Based Guidelines for FAIR Data and Metadata Management
}

\author{
Stefania Savoi $\left.{ }^{1}{ }^{(}\right)$, Panagiotis Arapitsas ${ }^{2, *}{ }^{\circledR}$, Éric Duchêne ${ }^{3}$, Maria Nikolantonaki ${ }^{4}{ }^{\circledR}$, Ignacio Ontañón ${ }^{5} \mathbb{C}^{(}$, \\ Silvia Carlin ${ }^{2}{ }^{-}$, Florian Schwander ${ }^{6}{ }^{(D}$, Régis D. Gougeon ${ }^{4}\left(\mathbb{D}\right.$, António César Silva Ferreira ${ }^{7}$, \\ Georgios Theodoridis ${ }^{8}{ }^{(0}$, Reinhard Töpfer ${ }^{6}\left(\mathbb{D}\right.$, Urska Vrhovsek $^{2}$, Anne-Francoise Adam-Blondon ${ }^{9}{ }^{\oplus}$, \\ Mario Pezzotti ${ }^{10, *}$ and Fulvio Mattivi ${ }^{2,11}$
}

Citation: Savoi, S.; Arapitsas, P.; Duchêne, É.; Nikolantonaki, M.; Ontañón, I.; Carlin, S.; Schwander, F.; Gougeon, R.D.; Ferreira, A.C.S.; Theodoridis, G.; et al. Grapevine and Wine Metabolomics-Based Guidelines for FAIR Data and Metadata Management. Metabolites 2021, 11, 757. https://doi.org/10.3390/ metabo11110757

Academic Editor: Jose Manuel Lorenzo Rodriguez

Received: 29 September 2021 Accepted: 30 October 2021

Published: 3 November 2021

Publisher's Note: MDPI stays neutral with regard to jurisdictional claims in published maps and institutional affiliations.

Copyright: (c) 2021 by the authors. Licensee MDPI, Basel, Switzerland. This article is an open access article distributed under the terms and conditions of the Creative Commons Attribution (CC BY) license (https:// creativecommons.org/licenses/by/ $4.0 /)$.
1 UMR AGAP, Montpellier University, CIRAD, INRAE, Institut Agro-Montpellier SupAgro, 34060 Montpellier, France; savoi.stefania@gmail.com

2 Department of Food Quality and Nutrition, Edmund Mach Foundation, Research and Innovation Centre, Via Edmund Mach 1, 38010 San Michele all'Adige, Italy; silvia.carlin@fmach.it (S.C.); urska.vrhovsek@fmach.it (U.V.); fulvio.mattivi@unitn.it (F.M.)

3 SVQV, University of Strasbourg, INRAE, F-68000 Colmar, France; eric.duchene@inrae.fr

4 UMR PAM Université de Bourgogne/Agro Sup Dijon, Institut Universitaire de la Vigne et du Vin, Jules Guyot, F-21000 Dijon, France; maria.nikolantonaki@u-bourgogne.fr (M.N.); regis.gougeon@u-bourgogne.fr (R.D.G.)

5 Laboratorio de Análisis Del Aroma y Enología, Departamento de Química Analítica, Facultad de Ciencias, Instituto Agroalimentario de Aragón (IA2), Universidad de Zaragoza, Calle de Pedro Cerbuna 12, 50009 Zaragoza, Spain; ionta@unizar.es

6 Julius Kühn-Institute, Institute for Grapevine Breeding Geilweilerhof, D-76833 Siebeldingen, Germany; florian.schwander@julius-kuehn.de (F.S.); reinhard.toepfer@julius-kuehn.de (R.T.)

7 CBQF-Centro de Biotecnologia e Química Fina.—Laboratório Associado, Escola Superior de Biotecnologia, Universidade Católica Portuguesa, Rua de Diogo Botelho, 1327, 4169-005 Porto, Portugal; asferreira@porto.ucp.pt

8 Laboratory of Analytical Chemistry, Department of Chemistry, Aristotle University of Thessaloniki, 54124 Thessaloniki, Greece; gtheodor@chem.auth.gr

9 Université Paris-Saclay, INRAE, URGI, 78026 Versailles, France; anne-francoise.adam-blondon@inrae.fr

10 Department of Biotechnology, University of Verona, 37134 Verona, Italy

11 Department of Cellular, Computational and Integrative Biology, CIBIO, University of Trento, 38123 Trento, Italy

* Correspondence: panagiotis.arapitsas@fmach.it or panagiotis.arapitsas@gmail.com (P.A.); mario.pezzotti@univr.it (M.P.)

Abstract: In the era of big and omics data, good organization, management, and description of experimental data are crucial for achieving high-quality datasets. This, in turn, is essential for the export of robust results, to publish reliable papers, make data more easily available, and unlock the huge potential of data reuse. Lately, more and more journals now require authors to share data and metadata according to the FAIR (Findable, Accessible, Interoperable, Reusable) principles. This work aims to provide a step-by-step guideline for the FAIR data and metadata management specific to grapevine and wine science. In detail, the guidelines include recommendations for the organization of data and metadata regarding (i) meaningful information on experimental design and phenotyping, (ii) sample collection, (iii) sample preparation, (iv) chemotype analysis, (v) data analysis (vi) metabolite annotation, and (vii) basic ontologies. We hope that these guidelines will be helpful for the grapevine and wine metabolomics community and that it will benefit from the true potential of data usage in creating new knowledge being revealed.

Keywords: open-data; plants; omics; Vitis; wine; metabolites; chromatography; mass spectrometry

\section{Introduction}

Thanks to the increasing availability of thousands of sequenced plant genomes [1], and the parallel uses of high-throughput analyses of next-generation sequencing techniques, 
such as the popular RNA sequencing [2], profiling the entire plant transcriptome and performing studies on gene expression during development and/or in response to biotic and environmental conditions [3] is now quite straightforward. In the past few years, the advancement of the methodologies based on liquid and gas chromatography (LC and GC) coupled with mass spectrometry (MS) and nuclear magnetic resonance (NMR) spectrometry opened a new field of research, metabolomics [4-6], that makes it possible to perform largescale measurements of hundreds or even thousands of metabolites in one run with targeted or untargeted approaches [7]. However, due to the chemical complexity of the metabolome, which differs from the four nucleotides with similar chemical properties that characterize the transcriptome, it is currently not possible to profile the entire plant metabolome using a single extraction protocol and a single analytical technique. The metabolomic space covered by an untargeted approach method can vary according to the analytical system, from dozens of major compounds in a NMR experiment $[8,9]$ to several hundreds or thousands of compounds for HRGC-MS [10] or HPLC-MS experiments [11]. Moreover, ultrahigh-resolution mass spectrometry (Fourier-transform ion cyclotron resonance mass spectrometry, FT-ICR-MS) [12,13] makes it possible to record thousands of signals for metabolic fingerprinting. Targeted approach methodologies usually cover a few to several dozen known metabolites [14]. Very often, therefore, several different protocols need to be integrated to achieve the desired coverage of the metabolome within a single study $[15,16]$. Moreover, the data are presented in a myriad of formats. Data from targeted experiments are quantitative and can be expressed as milliequivalents of multiple reference standards, with reference to the fresh or dry weight, etc. The data from untargeted experiments are the results of a process where the analyzed metabolome is not defined a priori, and this includes several unknown metabolites. Different sample preparation, instrumental analysis, and data analysis protocols will deliver complementary (but not conflicting) datasets and, therefore, possibly slightly different conclusions. This higher complexity requires highly organized data and metadata management, and metabolomic data must be combined with a detailed set of metadata to be correctly read and reused outside the original experiment.

With the recent developments at the level of LC-MS and GC-MS instrumentation (faster, more sensitive, more accurate, and with higher resolution) and data analysis bioinformatics tools, the number of metabolomics applications in the field of grapevine and wine research is increasing exponentially. Many researchers from the grape and wine science have started to work with big omics and delivered, over the last two decades, a huge volume of interesting data and new knowledge. However, in contrast to the grapevine transcriptomic field, where uploading the raw data in public repositories is mandatory for publication, in metabolomics, this action is just recommended and is very rarely mandatory [17].

Nevertheless, submitting the raw data to ad-hoc public repositories has become necessary in order to manage this exponential increase in biological data and their related metadata. This is further confirmed by the complexity and the variability of the protocols in use by the different laboratories. Often experimental designs, sample preparation, and analytical protocols are only partially described, and the terminology or ontologies used are not harmonized. In 2007, Sumner and colleagues [18] set up the first general guidelines to describe a metabolomic experiment, and since then, several groups have improved these general rules in various specific fields, especially in biofluid metabolomes (e.g., [19,20]). However, there are not many specific guidelines for grapevine and wine metabolomics research, and in that respect, the aim of the Cost Action CA17111 INTEGRAPE consortium was to fill this gap.

Nowadays, a researcher has multiple public repositories to upload metabolite analysis raw files to. A few are specific to the field of metabolomics (e.g., MetaboLights and Metabolomics Workbench), whereas others collect data from all the fields (e.g., figshare). MetaboLights is an open-access, curated database for metabolomics experiments connected with their raw data and associated metadata [21,22]. The database is cross-species, 
cross-technique, and covers metabolite structures and their reference spectra as well as their biological roles, locations, and concentrations. It is part of the ELIXIR infrastructure [23], hosted by the European Bioinformatics Institute (EMBL-EBI), and is one of the recommended metabolomics repositories for several leading journals in the field. The Metabolomics Workbench [24] is another repository for metabolomics data and metadata, as well as metabolite standards, protocols with tutorials, training, and analysis tools. The availability of both data and metadata is of the utmost importance in order to be able to adhere to the FAIR principles [25] that make data, experiments, and results Findable, Accessible, Interoperable, and Reusable.

The goal of this work is to provide all researchers within the grapevine and wine science fields with specific guidelines to implement best practices, improve the quality, availability, and usefulness of the data and the associated metadata, and facilitate the indexing of the produced dataset. A second purpose is encouraging the grapevine and wine science community to follow these guidelines as much as possible and, finally, to harmonize the data and metadata resources into a common, shared, and user-friendly format.

\section{Methods}

Each study created on public repositories has a unique alphanumeric study ID (e.g., MTBLS000 or ST0000). The study ID cannot be modified, and it is needed when referencing the study in manuscripts or elsewhere, together with the relevant URL. Please note that the study ID can be used to retrieve the dataset in MetaboLights, but as it is only mentioned in the materials and method section, it is not sufficient to obtain the indexing of the dataset in the Data Citation Index (DCI) of Clarivate Web of Science, which would be desirable in order to connect any published paper to the FAIR data present in the public repository. In order to facilitate the automatic inclusion in the DCI, it is recommended to specifically insert this persistent, resolvable identifier in a citation in the manuscript when the data are published. This also means that the data must be uploaded well in advance of the submission of the manuscript.

A general overview of the key protocols for the organization of data and metadata, especially when the final goal is to upload such information and raw files in public repositories (e.g., MetaboLights) under the FAIR principles, is shown in Figure 1. In addition, detailed information is presented in the following paragraphs.

Study descriptors are the fingerprints of the study, and they may include information such as the authors' names and IDs, an abstract giving a brief overview, publication references, and a list of keywords for indexing purposes. One of the most critical sections representing metadata enabling reproducibility is a series of protocols used in the study. Usually, these include a sample collection and sample preparation protocol (Tables 1 and 2). Moreover, one or multiple protocols describing the analyses performed are necessary. An example is a protocol for liquid or gas chromatography, followed by a second for mass spectrometry (Table 3). Other protocols are related to the steps taken for data transformation and, lastly, a protocol containing how the metabolites were annotated and quantified (Table 4). Finally, to adhere to the FAIR principle, the raw data, possibly in an open format, needs to be uploaded and linked to a study with the aim that other researchers can access it. 


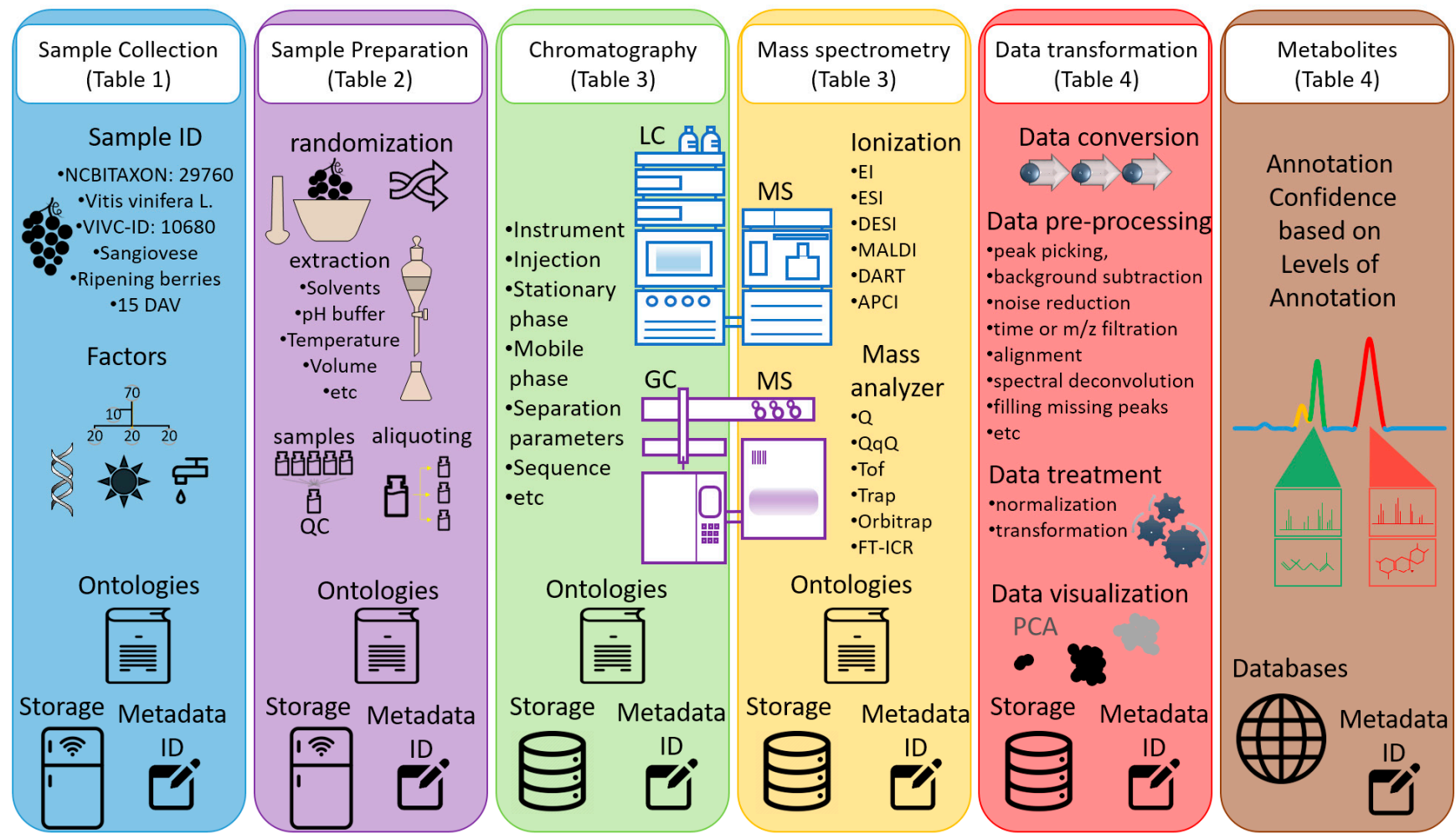

Figure 1. Overview of the required data and metadata management steps supplied with basic information on grapevine phenotyping and chromatography mass spectrometry techniques. Detailed information is included in each table.

Table 1. Sampling protocol (plant materials or wine sample).

\begin{tabular}{|c|c|}
\hline Field & Description \\
\hline Source & $\begin{array}{l}\text { Where the samples were collected. The use of an ID is recommended (https:/ / ror.org/, accessed on } \\
30 \text { October 2021). Example: Fondazione Edmund Mach collection (ID 0381bab64), or experimental } \\
\text { winery, winery, supermarket, etc. }\end{array}$ \\
\hline Organism & $\begin{array}{l}\text { An identifier for the organism at the species level. The use of the NCBI taxon ID is recommended. For } \\
\text { Vitis vinifera the ID is 29760. (https:/ / www.ncbi.nlm.nih.gov/taxonomy/, accessed on } 30 \text { October 2021). }\end{array}$ \\
\hline Specie(s) & $\begin{array}{l}\text { According to the standard scientific nomenclature, species name (formally: specific epithet) for the } \\
\text { organism under study (e.g., Vitis vinifera L.). }\end{array}$ \\
\hline Intraspecific name(s) & $\begin{array}{l}\text { Three field codes might be necessary to identify the exact plant material used in an experiment. } \\
\text { Field 1: code for the institution. Please refer to WIEWS codes from the FAO } \\
\text { (http:/ /www.fao.org/wiews/en/, accessed on } 30 \text { October 2021) or ROR codes (https:/ / ror.org, accessed } \\
\text { on } 30 \text { October 2021) for research organizations. } \\
\text { Field 2: type of plant material. The most commonly used denomination for grapevine material is the } \\
\text { variety name. We recommend using a standard name, such as the "prime name" extracted from the } \\
\text { VIVC database (http:/ /www.vivc.de, accessed on } 30 \text { October 2021). The type of plant material can be } \\
\text { classified with (i) the five-digit VIVC code for identified varieties, (ii) "PRO" for genotypes from } \\
\text { bi-parental crosses, (iii) "TL" for transgenic lines, (iv) "ESL" for lines regenerated from anthers or } \\
\text { somatic tissues, or (v) nothing when the type of plant material is not characterized. } \\
\text { Field 3: code used to identify the accession available in the institute. For plants from genetic resources, } \\
\text { the unique accession number of the EU-Vitis Database (http:/ / www.eu-vitis.de/, accessed on } \\
\text { 30 October 2021) is recommended. } \\
\text { Examples: FRA038_VIVC10077_274Col49 for Riesling clone number } 49 \text { available at INRAE Colmar. } \\
\text { FRA038_PRO_41207Col0011E for a genotype in the progeny from a cross between Riesling and } \\
\text { Gewürztraminer. DEU098-1980-315 for a specific Riesling accession in the Vitis collection of } \\
\text { JKI Geilweilerhof. }\end{array}$ \\
\hline
\end{tabular}


Table 1. Cont.

Field

Developmental stages

Tissue harvestin
method

Register the details about how the sampling occurred in the field/vineyard. For example, report if the samples were directly frozen and how (e.g., liquid $\mathrm{N}_{2}$, dry ice, freeze clamping, etc.), the date and time of collection, the place of collection, if samples were washed to remove unwanted external components (e.g., soil), shipping time and temperature, and sample storage before further preparation (e.g., $-80^{\circ} \mathrm{C}$ for two weeks).

Include information about the harvest date and period, if it was made manually or mechanically, the time of the day (morning, afternoon, night), grape sanitary status, crop yields, crushing and pressing devices and settings, yield of must or wine, pre-fermentative processing (e.g., grape cooling, sulfitation, etc.), information related to the experiment, etc.

Harvest protocol

Describe at which point in the production line the samples were collected (must, day of fermentation,

Sample Type (Wine) end of alcoholic fermentation, end of malolactic fermentation, after barrel aging, etc.).

Include information about additions (additives and processing aids, amount, timing, method of mixing), microorganism (source, genus, species, commercial name), must volumes, container type and volume, maceration technique, temperature, length of fermentation, use of oak/wood barrels (or wood staves or

Winemaking protocol chips), micro-oxygenation, first or second fermentation, disciplinary if PDO, and other information related to the experiment.

The following two documents from OIV can help:

https:/ / www.oiv.int/public/medias/4954/oiv-oeno-567a-2016-en.pdf (accessed on 30 October 2021). https://www.oiv.int/public/medias/7713/en-oiv-code-2021.pdf (accessed on 30 October 2021). 
Table 1. Cont.

\section{Field} analysis

Commercial wine

Packaging

Replicate sampling and analyses

\section{Storage conditions and \\ aliquoting prior to extraction}

Relocation or shipping info

Sample IDs list

\section{Description}

Include all available measurements (e.g., soluble solids, $\mathrm{pH}$, titratable acidity, fermentable nitrogen, sugars, ethanol, residual sugar, free and total sulfites, malic and lactic acid, total phenols, absorbance at 420 and $520 \mathrm{~nm}$, volatile acidity, $\mathrm{Cu}, \mathrm{Fe}$ ) and other information relevant for the experiment.

The next three documents from OIV can help:

https:/ / www.oiv.int/en/technical-standards-and-documents/methods-of-analysis/compendium-ofinternational-methods-of-analysis-of-wines-and-musts (accessed on 30 October 2021).

https:/ / www.oiv.int/en/technical-standards-and-documents / methods-of-analysis/the-guidelinesin-oenology (accessed on 30 October 2021).

https:/ / www.oiv.int/en/technical-standards-and-documents (accessed on 30 October 2021).

If the study is based on commercial wines sourced from the market, provide information about source (supermarket, wine shop, winery, etc.), date of sampling, price, PDO data, cultivar(s), winery, region, country, vintage, color, basic oenological analysis, lot number, bottling date, and other information related to the experiment.

Include information about packaging material (glass bottle, tetra-pak, metallic, plastic, etc.), packaging transparency (flint, green, amber, dark), volume, stopper (corks-natural, colmated, agglomerated, $1+1$, micro-agglomerated-, synthetic, screw caps, glass, etc.), and other information related to the experiment.

The number of samples represents the "sample size", and all the samples together represent the "sample set". A part of the "sample set", thus a "sample subset", can be used for the analysis. A minimum of triplicate $(n=3)$ biological sampling is proposed, with $n=5$ preferred. It is more critical to measure biological replicates than technical ones.

Report information about temperature, duration, possible additives, volumes, container, and atmosphere. Samples can be divided into small aliquots. Report method, volume or weight, and number of aliquots.

Provide information about the shipping conditions (if relevant).

Sample ID list connected to the above information.

Table 2. Extraction (sample preparation) protocol

\begin{tabular}{cl}
\hline Field & \multicolumn{1}{c}{ Description } \\
\hline Randomization & $\begin{array}{l}\text { Report if the sample preparation order was randomized and how (https://www.random.org/sequences/, accessed } \\
\text { on 30 October 2021). }\end{array}$ \\
\hline Extraction parameters & $\begin{array}{l}\text { Solvent(s), pH and ionic strength of the buffer, solvent temperature and volume(s) per quantity of tissue, internal } \\
\text { standard(s), number of replicate extracts (technical and biological replicates), sequential extraction, and } \\
\text { extraction time. }\end{array}$ \\
\hline Concentration/Dilution & Extract concentration, dilution, and resolubilization processes (e.g., dried under nitrogen, solubilized in methanol). \\
\hline Enrichment & Extract enrichment (e.g., solid-phase extraction, desalting, molecular cut-off, ion exchanges, rotary vapor). \\
\hline Extract treatments & Extract cleanup and/or use of additives (e.g., ultrafiltration, centrifugation, the addition of antioxidants, pH change). \\
\hline Derivatization & Report the protocol of derivatization (the chemical used, temperature, time, etc.). \\
\hline Quality Control Sample(s) & $\begin{array}{l}\text { Report if a QC pooled sample was prepared using extracts of the entire "sample set" or a "sample subset". In addition, } \\
\text { report the method (volume or weight from each sample and total amount of the QC pooled sample). }\end{array}$ \\
\hline Reference Material & $\begin{array}{l}\text { Report if any biological reference material and/or a standard mixture was used and how it was purchased or } \\
\text { prepared. This material can also be used as QC samples. }\end{array}$ \\
\hline Blanks & Report how the blank sample was prepared. \\
\hline Aliquoting & Aliquots prepared during or after the sample preparation (code, volume, number). This includes the QC samples. \\
\hline Storage-Relocation & $\begin{array}{l}\text { Extract storage (e.g., temperature, duration, atmosphere, volumes, containers, etc.) and/or relocation (e.g., } \\
\text { temperature, duration, atmosphere, places). }\end{array}$ \\
\hline Internal standard(s) \\
addition
\end{tabular}$\quad$\begin{tabular}{l} 
Internal standard(s) at any stage(s). \\
\hline $\begin{array}{l}\text { Samples ID list } \\
\text { Update the Sample ID list, including the names or the IDs of the extracts. Often more than one extraction protocol is } \\
\text { applied to the same samples. }\end{array}$ \\
\hline
\end{tabular}


Table 3. Chromatography and Mass spectrometry protocol.

\begin{tabular}{|c|c|}
\hline Field & Description \\
\hline Instrument & $\begin{array}{l}\text { Manufacturer, model number, software package and version. The majority of the instruments can be found in the } \\
\text { EMBL/EBI ontology (https://www.ebi.ac.uk/ols/ontologies/ms, accessed on } 30 \text { October 2021). If this is the case, we } \\
\text { recommend the use of the ontologies; if not, use free text. }\end{array}$ \\
\hline Injection & $\begin{array}{l}\text { Auto-injector (manufacturer, model, type, software, injector/loop volume, wash cycles, solvents, volume, SPME } \\
\text { parameters, automatic derivatization, injector temperature, split or splitless mode, and ratio, etc.). }\end{array}$ \\
\hline Stationary phase & $\begin{array}{l}\text { Separation column(s) and pre/guard column (manufacturer, model/name, stationary phase composition, particles, } \\
\text { internal diameter, physical parameters, length, parameters of 2D chromatography, etc.). }\end{array}$ \\
\hline Mobile phase & $\begin{array}{l}\text { Mobile phase (e.g., gases, solvents, buffers, } \mathrm{pH} \text { ) including their preparation protocol (information of the type of flasks, } \\
\text { pipette, degasser, etc.) and post-column modifiers (if applied). }\end{array}$ \\
\hline Separation & $\begin{array}{l}\text { Separation parameters (sample temperature, mobile phases composition(s), gradient profile, column temperature, flow } \\
\text { rate(s), pressure, etc.). }\end{array}$ \\
\hline Sequence & $\begin{array}{l}\text { Sequence duration and length of stay of the sample in the sampler before analysis. Report if the "sample set" or "sample } \\
\text { subset" order was randomized and the frequency of the QC analysis (all types of QC samples used). }\end{array}$ \\
\hline $\begin{array}{l}\text { Sample introduction } \\
\text { and delivery }\end{array}$ & Direct infusion (continuous or not) after GC, CE, or LC separation. \\
\hline Ionization source & $\begin{array}{l}\text { Ionization mode (EI, APCI, ESI, etc.), polarity (positive or negative), vacuum pressure, skimmer/focusing lens voltages } \\
\text { (e.g., capillary voltage, etc.), gas flows (e.g., nebulization gas, cone gas, source temperature, etc.). }\end{array}$ \\
\hline Mass analyzer & $\begin{array}{l}\text { Type of analyzer (e.g., quadrupole, ion-trap, time-of-flight, FT-ICR, including combinations of these for hybrid } \\
\text { instruments). The majority of the analyzers can be found in EMBL/EBI ontology } \\
\text { (https://www.ebi.ac.uk/ols/ontologies/ms, accessed on } 30 \text { October 2021). }\end{array}$ \\
\hline $\begin{array}{l}\text { Acquisition mode and } \\
\text { parameters }\end{array}$ & $\begin{array}{l}\text { For a single quadrupole instrument, the scan modes are full scan and sim; for a triple quadrupole instrument, common } \\
\text { modes are full scan, product scan, precursor scan, neutral loss scan and MRM. } \\
\text { In high-resolution MS (QTof and Orbitrap), common scan modes are: (a) full scan; (b) data-dependent acquisition, such } \\
\text { as MS/MS; and c) data-independent acquisition, such as Swath, Sonar, MSall, MSn, MSe, MSc2, AIF-MS2, vDIA, bbCID. } \\
\text { All the parameters of the acquisition mode should be reported, such as the m/z scan range, polarity(ies), scan speed, } \\
\text { collision energy(ies), cycle time, resolution, mass accuracy, and spectral acquisition rate, vacuum pressure, various } \\
\text { voltages, etc. }\end{array}$ \\
\hline Ion Mobility & Type (DTIMS, TIMS, DMS, etc.), place (e.g., before or after the quadrupole), buffer gas, separation parameters. \\
\hline $\begin{array}{l}\text { Technique-specific } \\
\text { sample preparation }\end{array}$ & $\begin{array}{l}\text { Re-suspension of sample (e.g., in MeOH:water 1:1 with } 0.2 \% \text { formic acid), derivatization, volume injected, and internal } \\
\text { calibrant(s) added (if relevant). }\end{array}$ \\
\hline Calibration & Calibration compound(s) and mode. \\
\hline Lock spray & Concentration, lock mass, flow rate, and frequency. \\
\hline Analysis or Assay ID & $\begin{array}{l}\text { Update the Sample ID list, including the names or the IDs of the raw assay files. This name could include the date of the } \\
\text { analysis, the order of the analysis, and information about the protocol. The sample with the ID: SAN12 could have as } \\
\text { Assay ID: 20201214_055_SAN12_RP_NEG, where the first part gives information about the date of analysis, the second } \\
\text { the order of the analysis, the third the sample ID, and the fourth about the chromatography protocol. }\end{array}$ \\
\hline
\end{tabular}

Table 4. Data transformation/conversion parameters protocol and metabolite identification.

\begin{tabular}{cl}
\hline \multicolumn{1}{c}{ Field } & \multicolumn{1}{c}{ Description } \\
\hline $\begin{array}{c}\text { Raw data } \\
\text { format }\end{array}$ & Report the format of the original raw data, as registered by the instrument and its software. \\
\hline Data conversion & $\begin{array}{l}\text { Often the raw data are converted to "open" (or not) formats, such as net.CDF, XML, MZml, etc., for their further analysis. } \\
\text { Report the software and its version used for the data conversion and the parameters used. }\end{array}$ \\
\hline Data pre-processing & $\begin{array}{l}\text { The original or the converted data are often processed before the statistical analysis. For the MS data, the process might } \\
\text { include peak picking, background subtraction, noise reduction, time or } m / z \text { filtration, alignment, spectral deconvolution, } \\
\text { smoothing, binning, data reduction, filling missing peaks, etc. Report the software and its version used together with the } \\
\text { parameters. The most popular software are MZmine, XCMS, MSdial, metaMS, Progenesis QI, and MetAlign. }\end{array}$ \\
\hline Data treatment & $\begin{array}{l}\text { The obtained peak table from the data pre-treatment can be further treated with normalization and scaling tools. First, } \\
\text { report the software, its version, and the parameters used. Then, inspecting the data for drift correction or outliers' } \\
\text { detection is envisaged. }\end{array}$ \\
\hline $\begin{array}{c}\text { Annotation } \\
\text { confidence }\end{array}$ & $\begin{array}{l}\text { The correct peak or metabolite annotation is crucial for the interpretation of the results, and it is important to provide } \\
\text { information as far as the confidence of each annotation will allow by applying one of the below-listed annotation } \\
\text { level protocols. }\end{array}$ \\
\hline
\end{tabular}


Table 4. Cont.

Field annotation [18]

Five levels annotation [31]

This is the most common method used to report the annotation confidence in metabolomics. It includes the following levels of annotation:

1. Identified compounds. A minimum of two independent and orthogonal data relative to an authentic compound analyzed under identical experimental conditions is proposed as necessary to validate non-novel metabolite identifications (e.g., retention time/index and mass spectrum, retention time and NMR spectrum, accurate mass and tandem MS, accurate mass and isotope pattern, full ${ }^{1} \mathrm{H}$ and / or ${ }^{13} \mathrm{C}$ NMR, 2-D NMR spectra).

2. Putatively annotated compounds. This level is applied when the annotation is made without chemical reference standards, based upon physicochemical properties and/or spectral similarity with public/commercial spectral libraries or literature. If spectral matching is utilized in the identification process, then the authentic spectra used for the spectral matching should be described appropriately or libraries made publicly available.

3. Putatively characterized compound classes. The annotation is based upon characteristic physicochemical properties of a chemical class of compounds or by spectral similarity to known compounds of a chemical class (e.g., hexose, carotenoid, lipid, anthocyanin, etc.).

4. Unknown compounds. Although unidentified or unclassified, these metabolites can still be differentiated and quantified based upon spectral data.

This is the second most used method to report the annotation confidence in metabolomics. It includes the following levels of annotation:

Level 1: Confirmed structure represents the ideal situation, where the proposed structure has been confirmed via appropriate measurement of a reference standard with MS, MS/MS and retention time matching. If possible, an orthogonal method should also be used.

Level 2: Probable structure indicates that it was possible to propose an exact structure using different evidence. For Level 2a: a library that involves matching literature or library spectrum data where the spectrum-structure match is unambiguous. Care is needed when comparing spectra recorded with different acquisition parameters (e.g., resolution, collision energy, ionization, MS level, retention behavior) to ensure the validity of the match; decision criteria should be clearly presented. For Level 2b: diagnostic represents the case where no other structure fits the experimental information, but no standard or literature information is available for confirmation. Evidence can include diagnostic MS/MS fragments and/or ionization behavior, parent compound information, and the experimental context.

Level 3: Tentative candidate(s) describes/e a "grey zone", where evidence exists for possible structure(s), but the information for one exact structure only is insufficient (e.g., positional isomers).

Level 4: Unequivocal molecular formula is possible when a formula can be unambiguously assigned using the spectral information (e.g., adduct, isotope, and/or fragment information), but insufficient evidence exists to propose possible structures. The MS/MS could be uninformative, contain interferences, or not even exist.

Level 5: Exact mass $(\mathrm{m} / \mathrm{z})$ can be measured in a sample and be of specific interest for the investigation but lack information to assign even a formula. Screening and nontarget methods allow the tracing of these masses in other investigations, but level 5 indicates that no unequivocal information about the structure or formula exists. It is even possible to record the MS/MS of a level 5 mass and save it as an "unknown" spectrum in a database. This level should only apply to a few masses of specific interest since it would be counterproductive to label all masses in a sample as level 5 . Blank measurements should be used to ensure the substance does not arise from sample preparation or measurement.

The metabolomics community recently released a new method by introducing subclasses (A-F) for unambiguous metabolite annotation. https://drive.google.com/file/d/1PJLdPCkz8ymX8SgZ4W15Sw4ZG-dlyWWU/view, accessed on 30 October 2021.

The proposed levels are:

A: Known enantiomer. A single defined enantiomer or a single defined achiral metabolite. Molecular formula, structure, and stereochemistry, including chirality, are known. Usually requires isolation of metabolite and complete structure determination or chiral chromatography on metabolite in a mixture to prove chirality and matching of two orthogonal pieces of data with an authentic chemical standard. For achiral metabolites, it requires the matching of two orthogonal pieces of data with authentic chemical standards (e.g., RT and MS/MS mass spectrum).

B: Known diastereomer. One of two enantiomers. Known molecular formula, structure, and stereochemistry but unknown chirality. Requires matching of two orthogonal pieces of data with authentic chemical standards (e.g., RT and MS/MS mass spectrum).

Metabolomics Society's Metabolite Identification Task Group
C: Known structure/DB position. One of a number of stereoisomers, e.g., E/Z geometric or cis-/trans-ring isomers. Known molecular formula and structure but unknown stereochemistry. Requires matching of two orthogonal pieces of data with authentic chemical standards (e.g., RT and MS/MS mass spectrum).

D: Known functional group. One of a number of positional isomers. Known molecular formula and metabolite class but unknown structure, e.g., high-resolution mass spectrometry provides unique and unambiguous single molecular formula, and additional data proves metabolite class membership.

E: Known formula. One of a number of possible compounds of known molecular formula. Known molecular formula but unknown structure, e.g., high-resolution mass spectrometry provides the unique and unambiguous single molecular formula.

F: Known structural class. Specific spectral features defining a structural class. Unknown molecular formula but a known class of metabolite; characteristic signals of metabolite class in the sample.

G: Known formula. Specific spectral futures. Unknown molecular formula; characteristic signals of unknown metabolite in the sample.

Identify the annotated metabolites with a unique identifier (ID) corresponding to one of the following databases:
Chemical entities of biological interest (ChEBI), HMDB, FoodDB, KEGG, Chemspider, PubChem, COlleCtion of Op

Metabolite ID Natural ProdUcTs ID, or CAS. 


\subsection{Sample Collection}

Within the protocol adopted in this study, it is essential to describe the origin of the samples (source, organism, genus, species, intraspecific name, organism part, etc.), to include some technical description of the experiments (row and plant spacing, rootstock, planting date, training system, soil management technique, etc.), any relevant treatments, biotic or abiotic stresses; time points or cultural operations, such as pruning, hedging, fertilization, pesticide spraying, etc., and the collection and storage procedure (Table 1). Moreover, information on how to describe experiments involving wine samples is reported. We highly recommend the use of OIV (International Organization of Vine and Wine) terminologies, ontologies, and standards. Other necessary metadata on the experimental design are the developmental stage, phenological description, geographic location, agronomical practices, etc. A set of recommendations on adequately describing a grapevine experiment to guarantee interoperability and standard ontologies between different datasets is based on the international metadata standard for Plant Phenotyping experiments (MIAPPE, www.miappe.org, accessed on 30 October 2021) [26]. The MIAPPE checklist is a pdf file that lists all the items recommended to describe an experiment, from the general characteristics of an investigation/study to the biological samples proposing descriptors for experimental factors and environmental variables.

\subsection{Extraction (Sample Preparation)}

This protocol is intended to describe any extraction or sample preparation method applied to the sample before analysis (Table 2). It is very important to report information on any quality control samples (QC) prepared for the assay, e.g., pooled samples, blanks, standards mixtures, etc. QCs are used to handle any analytical variability, for example, by monitoring the performances of the instrument, any shifts in compounds retention times, signal intensity, to reduce noise, to correct for batch effect or systematic errors, and they should be injected at the beginning of the experiment and then repeatedly every 5 to 10 samples, depending on the size of the sample set. The pooled QC samples should represent the overall composition of all the samples, encompassing all the major developmental stages or treatments of the samples under analysis, and they should be prepared and extracted in the same way as the other samples. Blanks are another type of QC, which should be prepared using the same protocol of the study, except that the sample matrix is to be replaced by nothing, if it is solid, or by the extraction solvent (or water), if it is liquid. Blanks will help find any artifacts, contamination, or unwanted by-products. One or more internal standards can be added to the real samples, blanks, and pooled samples, which can be helpful during the normalization process. Randomization of the sample extraction/sample preparation and injection order is also encouraged to help in reducing bias in the interpretation of the results. In particular, a systematic scheme for the sample run order should be created by combining the experimental design with complete or group-wise randomization [19]. Complete randomization is the preferred solution, but partial randomization is also acceptable in case of a large number of samples to be analyzed.

\subsection{Chromatography and Mass Spectrometry}

By definition, chromatography is an analytical, separative technique where compounds are eluted in different retention times, based on molecular characteristics and interaction type, that uses ion exchange, surface adsorption, partition, and size exclusion mechanisms. For reasons of reproducibility, it is necessary to provide details of the instrument and column(s), mobile phase and gradient, and settings, such as temperatures, flow rate, injection volume, etc. (Table 3). Moreover, the chemical methods ontology (https:/ / www.ebi.ac.uk/ols/ontologies/chmo, accessed on 30 October 2021) can help find vocabularies to describe the methods and tools used to collect data in chemical experiments, such as chromatography and mass spectrometry. 
A mass spectrometer measures the abundance of the mass-to-charge ratio $(\mathrm{m} / \mathrm{z})$ of the ions generated by the ionization process of the eluted metabolites, previously separated by GC or LC. Within this protocol, it is mandatory to provide details of the instrument used, ion source, ionization mode (positive/negative), $\mathrm{m} / \mathrm{z}$ range, and specific parameters such as temperatures, voltages, flow rates, scan rates, etc. (Table 3). Since the analyzed metabolites are not predefined in metabolomics, method validation is rather tricky. However, a minimum reporting of instrumental performance parameters is encouraged. For example, the description of the nature and method(s) used is essential to ensure instrumental sensitivity, selective, linearity, stability, resolution, and mass accuracy. The QC samples distribution in a PCA plot is a good indicator.

These guidelines can be applied even in the case of less extensive experimental settings and methodologies, such as targeted chromatography or spectroscopy (e.g., FTIR Fouriertransform infrared spectroscopy) and beyond (e.g., genomic approaches), especially for sample collection, classification, preparation, and data processing, which are supposed to be reported with the same level of detail.

\subsection{Data Transformation and Metabolite Identification}

The data acquired by the instrument need to be converted and processed before any further data and statistical analysis. In order to obtain a raw data matrix, features $(\mathrm{m} / \mathrm{z}$ intensity related to a specific retention time) need to be extracted, often with an automatic pipeline or thanks to dedicated commercial software. Hence, this protocol requires the details of the methods, pipeline, or software used to transform the raw data and all the steps and parameters used to extract the features (Table 4). More helpful information can also be found in [19], Appendix 4.

Metabolite identification is one of the most critical phases in metabolomics, requiring expertise, and is a time-consuming task. In the case of a targeted method, features can be identified by means of reference standards or library matching and quantified by their calibration curve. The identification of unknown features extracted from untargeted methods is more challenging, although the availability of several databases might help in this task $[19,20]$. In Table 4, we describe the three main methods used for metabolite annotation confidence level. It is mandatory to use one of them in reporting the results because the use of common names is often confusing.

\section{Results}

To the best of our knowledge, up to September 2021, we were able to retrieve fourteen publicly available grapevine metabolome datasets $[15,16,32-39]$ and seven others related to wine science [40-46], with the majority of such studies deposited in the repository MetaboLights. It is foreseeable that the number will quickly increase in the near future as the application of metabolomics to plant science increases. These guidelines are intended to assist the grapevine and wine metabolomics community in publishing their data in compliance with the FAIR protocol.

In MTBLS2876 [32,47], there are data and metadata on the most important classes of grapevine leaf metabolites (primary compounds, lipids, phenols, and volatile organic compounds) detected at several time points after artificial inoculation of the oomycete Plasmopara viticola in resistant and susceptible grape varieties.

MTBLS784 [33,48] reported a study on the pre-harvest application of methyl jasmonate on Vitis labrusca grapes to verify the interaction with the phenolic compounds content by UPLC-HRMS-QTOF.

MTBLS968 [34,49] is a study about the accumulation profiles of terpene metabolites in three Muscat table grape cultivars through HS-SPME-GCMS.

The following three datasets (MTBLS898, MTBLS982, MTBLS984) [16,50-52] comprehensively characterized the metabolic response of Merlot grape berries exposed to water deficit at different developmental stages by UPLC-MS/MS, SPME-GC-MS, and UPLC-DAD assays for the analysis of phenolic, volatiles and carotenoids compounds. 
The MTBLS392 study [35,53] aimed to explore the core microbiota and metabolome of Vitis vinifera L. cv. Corvina grapes and musts during different vintages, by two-dimensional gas chromatography time-of-flight mass spectrometry.

Studies MTBLS897, MTBLS892, and MTBLS889 [15,54-56] represent the datasets of broad research investigating the impact of water deficit on the secondary metabolism of white grapes using large scale metabolites analyses in a season characterized by prolonged drought.

The study with ID MTBLS346 [36,57] investigated the downstream metabolic changes of Silcora and Thompson seedless grape cultivars when genetically modified through the insertion of the DefH9-iaaM gene into their genome. The effect of the genetic modification upon the grape metabolome was evaluated by ${ }^{1} \mathrm{H}-\mathrm{NMR}$ and exploratory data analysis and chemometrics methods.

MTBLS209 [37,58] is a comparative study of the metabolome of the three grape berry tissues (skin, pulp, seeds) of three American Vitis species (Vitis cinerea, Vitis californica, Vitis arizonica) together with four interspecific hybrids, and seven Vitis vinifera cultivars.

The authors of the MTBLS85 study [38,59] presented an automated data analysis of high throughput high-performance liquid chromatography with diode array detection (HPLC-DAD) data using multivariate curve resolution; a case study on the stability of isoprenoids in grape extracts under two different experimental regimes serves to illustrate the potential of the method.

And, finally, study MTBLS39 $[39,60]$ described the plasticity of the berry metabolome of the cultivar Corvina sampled during development at different geographical locations.

Concerning wine metabolomics, study MTBLS1677 [40,61] analyzed a set of 917 wines of Czech origin using nuclear magnetic resonance spectroscopy with the aim to build and evaluate multivariate statistical models and machine learning methods for the classification of such wines based on type, variety, and location.

The aim of the next project, with the raw data and metadata deposited with ID MTBLS1443 [41,62], was to register the metabolome of 11 single-cultivar single-vintage Italian red wines from 12 regions across Italy, each one produced in their terroirs under ad hoc legal frameworks in a LC-MS-based untargeted single-batch analysis, to guarantee their quality and origin.

In MTBLS2330 [42,63], the authors aimed to study the impact of water deficit on the concentration of key flavor and phenolic secondary metabolites of both red and white wines, linking the previously observed drought-induced grape compositional changes to the wines.

Study MTBLS212 $[43,64]$ untangled the wine metabolome by combining untargeted SMPE-GCxGC-TOF-MS and sensory analysis to profile Sauvignon blanc co-fermented with seven different yeasts.

The MTBLS137 dataset $[44,65]$ reported the UPLC-QTof MS untargeted analysis of Vitis vinifera L. leaves, collected in Italy and Germany from two fungus-resistant grape varieties (PIWI), Regent and Phoenix. It is an illustrative example of the use of the MetaDB pipeline. MetaDB was developed in order to combine with a user-friendly web-based, different bioinformatic tool used in metabolomics, which takes care of (a) metadata organization, (b) creation of randomized sequences including QC sample, (c) data quality evaluation, (d) data storage organization, (e) data analysis, and (f) submission to public repositories.

MTBLS55 $[45,66]$ is a study about the influence of storage condition and duration on the "chemical age" of red wines.

And finally, study ST000006 [46,67], deposited at Metabolomics Workbench repository, reported the data of a GC-TOF analysis on seventeen white wines for investigating the chemical basis for mouthfeel properties in wine.

\section{Discussion}

Although limited in number, the above-described public datasets cover a big part of the technological and scientific themes of the grapevine and wine community. Their most 
common aim was to investigate the cultivar/geographical differentiation, and second, to study the abiotic and biotic stresses. However, considering the scientific literature, there are still many fields that are widely present in the literature but are not covered by these datasets, from viticulture practices (rootstock, training systems, soil, leaf removal, etc.) to winemaking techniques (enzymes/microorganism/coadjuvant, physical technologies, special wines, etc.) and shelf life (packaging, exposure to oxygen, light, temperature, and the evolution during storage, etc.). It is positive to see that the above-described datasets retrieved from public repositories were produced using an array of diverse, modern, or traditional instrumental/analytical techniques, such as gas/liquid chromatography, various mass spectrometers, nuclear magnetic resonance, and diode array.

Engaging in completely open science may be unrealistic and even undesirable for many researchers. However, the use of FAIR repositories is becoming mandatory by many funding agencies. We urge researchers working in viti-oenology to learn and adopt existing, robust tools that are immediately helpful and important to them in order to improve the visibility, quotability, and preservation of their data, which will be safely archived and ready to reuse.

And we all know, and it is especially true in the era of big data, that reproducibility and transparency are key tenets of the scientific process that requires reliability and generality of results and methods [68]. Of course, making a dataset publicly available is not enough since data and metadata management according to the FAIR principles is equally important. This holistic process could help make the studies easier to find for humans and computer tools (ontologies, IDs, rich and indexed metadata, etc.), accessible to all (open, free, and universally applicable protocols), interoperable with other (meta)data and datasets, and optimized to be reused and useful to other scientists. The connection between metabolomics and the other omics (genomics, transcriptomics, and proteomics) towards multi-omics, which for the moment is still in its infancy, will be easier and more straightforward. Research institutes without direct access to multi-scientific platforms will be less penalized and limited because they might have access to FAIR datasets and can build directly on primary work. The real potential of big data, which is impossible to explore in one study, will be unlocked. The reproducibility and transparency of the published knowledge will be improved due to the curation of the coded workflows (shared in public repositories) and revision (journal's peer revision). Metabolite annotation, which is now the bottleneck of most studies, will be substantially improved by systematically using metabolite IDs and reference spectra sharing and via access to existing, searchable databases for confirmation of the chemical features of interest in different experimental contexts.

On the other hand, we acknowledge that this process is often complex and timeconsuming and that guidelines, tutorials, and training are necessary. Since the general guidelines are often complicated and difficult to understand, it is necessary to support the research community and not work in isolation. COST CA17111 INTEGRAPE decided to write the first specific guidelines for grapevine and wine metabolomics FAIR data and metadata management.

It is encouraging to discover that the great majority of the publicly available metabolomic datasets in grapevine and wine science, and especially the studies uploaded to MetaboLights (Supplementary Materials Table S1), are filed under FAIR principles, even though some were released before the FAIR publication. They all include the appropriate protocols, the use of ontologies and IDs, and have raw files uploaded on the website. There is only one study on a different public repository, which lacks many important metadata parameters and does not include raw files (but an excel document), but this is one of the oldest ones since it was released in 2013. The long experience and the curation protocol of MetaboLights make this website an excellent hub for the metabolomics community. However, a better connection between MetaboLights, VIVC IDs, and MIAPPE ontologies is strongly suggested. 


\section{Concluding Remarks}

In the era of molecular system biology and integrative studies beyond single omics techniques, the availability of raw data supported with a rich set of metadata is now compulsory in all omics research. To advance in such specific fields, the research community requires data-sharing, common language, open science, and straight collaborations between the different scientific disciplines. It is time that the «metabolomics» scientists learn from the successful experience of their «genomics» brothers and start to manage, organize and share their data and metadata according to the FAIR principles.

Within this frame, the COST Action CA 17111 INTEGRAPE was launched to integrate data at different levels to maximize the power of omics in order to improve reproducibility, transparency, and accessibility. It does this by optimizing the data flows and interoperability between datasets, different analytical tools, data standards, and ontologies and by providing recommendations and tools with the final purpose of grapevine improvement. Therefore, we strongly promote these phenotypic and metabolomics/chemotypic guidelines to our grape and wine community, knowing that the most valuable experiment is the one already filed and made available on a dedicated repository online.

Supplementary Materials: The following are available online at https:/ / www.mdpi.com/article/10 $.3390 /$ metabo11110757/s1, Table S1: List of publicly available datasets on grape and wine metabolome and their adhesion to these guidelines.

Author Contributions: Conceptualization: S.S., P.A. and F.M.; writing—original draft preparation: S.S., P.A., É.D. and F.M.; writing-review and editing, S.S., P.A., É.D., M.N., I.O., S.C., F.S., R.D.G., A.C.S.F., G.T., R.T., U.V., A.-F.A.-B., M.P. and F.M. All authors have read and agreed to the published version of the manuscript.

Funding: This article/publication is based upon work from COST Action CA 17111 INTEGRAPE, supported by COST (European Cooperation in Science and Technology).

Institutional Review Board Statement: Not applicable.

Informed Consent Statement: Not applicable.

Data Availability Statement: Not applicable.

Acknowledgments: This article/publication is based upon work from COST Action CA 17111 INTEGRAPE, supported by COST (European Cooperation in Science and Technology). We would like to thank Nigel Robinson for his suggestions on the Data Citation Index, Clarivate.

Conflicts of Interest: The authors declare no conflict of interest.

\section{References}

1. Chen, F.; Song, Y.; Li, X.; Chen, J.; Mo, L.; Zhang, X.; Lin, Z.; Zhang, L. Genome Sequences of Horticultural Plants: Past, Present, and Future. Hortic. Res. 2019, 6, 112. [CrossRef] [PubMed]

2. Wang, Z.; Gerstein, M.; Snyder, M. RNA-Seq: A Revolutionary Tool for Transcriptomics. Nat. Rev. Genet. 2009, 10, 57-63. [CrossRef] [PubMed]

3. Massonnet, M.; Fasoli, M.; Vondras, A.M.; Zenoni, S.; Santo, S.D.; Vannozzi, A.; Castellarin, S.D.; Pezzotti, M.; Cantu, D. Grape transcriptomics and viticulture. In The Grape Genome; Cantu, D., Walker, M.A., Eds.; Compendium of Plant Genomes; Springer International Publishing: Cham, Switzerland, 2019; pp. 275-299, ISBN 978-3-030-18601-2.

4. Fiehn, O.; Kopka, J.; Dörmann, P.; Altmann, T.; Trethewey, R.N.; Willmitzer, L. Metabolite Profiling for Plant Functional Genomics. Nat. Biotechnol. 2000, 18, 1157-1161. [CrossRef] [PubMed]

5. Fiehn, O. Metabolomics-The Link between Genotypes and Phenotypes. Plant Mol. Biol. 2002, 48, 155-171. [CrossRef]

6. Alseekh, S.; Fernie, A.R. Metabolomics 20 Years on: What Have We Learned and What Hurdles Remain? Plant J. 2018, 94, 933-942. [CrossRef] [PubMed]

7. De Vos, R.C.; Moco, S.; Lommen, A.; Keurentjes, J.J.; Bino, R.J.; Hall, R.D. Untargeted Large-Scale Plant Metabolomics Using Liquid Chromatography Coupled to Mass Spectrometry. Nat. Protoc. 2007, 2, 778-791. [CrossRef] [PubMed]

8. Pereira, G.E.; Gaudillere, J.-P.; van Leeuwen, C.; Hilbert, G.; Maucourt, M.; Deborde, C.; Moing, A.; Rolin, D. 1H NMR Metabolite Fingerprints of Grape Berry: Comparison of Vintage and Soil Effects in Bordeaux Grapevine Growing Areas. Anal. Chim. Acta 2006, 563, 346-352. [CrossRef] 
9. Gougeon, L.; da Costa, G.; Guyon, F.; Richard, T. 1H NMR Metabolomics Applied to Bordeaux Red Wines. Food Chem. 2019, 301, 125257. [CrossRef]

10. Vrhovsek, U.; Lotti, C.; Masuero, D.; Carlin, S.; Weingart, G.; Mattivi, F. Quantitative Metabolic Profiling of Grape, Apple and Raspberry Volatile Compounds (VOCs) Using a GC/MS/MS Method. J. Chromatogr. B 2014, 966, 132-139. [CrossRef] [PubMed]

11. Vrhovsek, U.; Masuero, D.; Gasperotti, M.; Franceschi, P.; Caputi, L.; Viola, R.; Mattivi, F. A Versatile Targeted Metabolomics Method for the Rapid Quantification of Multiple Classes of Phenolics in Fruits and Beverages. J. Agric. Food Chem. 2012, 60, 8831-8840. [CrossRef] [PubMed]

12. Maia, M.; Monteiro, F.; Sebastiana, M.; Marques, A.P.; Ferreira, A.E.N.; Freire, A.P.; Cordeiro, C.; Figueiredo, A.; Sousa Silva, M. Metabolite Extraction for High-Throughput FTICR-MS-Based Metabolomics of Grapevine Leaves. EuPA Open Proteom. 2016, 12, 4-9. [CrossRef] [PubMed]

13. Romanet, R.; Sarhane, Z.; Bahut, F.; Uhl, J.; Schmitt-Kopplin, P.; Nikolantonaki, M.; Gougeon, R.D. Exploring the Chemical Space of White Wine Antioxidant Capacity: A Combined DPPH, EPR and FT-ICR-MS Study. Food Chem. 2021, 355, 129566. [CrossRef]

14. Figueiredo, J.; Cavaco, A.R.; Guerra-Guimarães, L.; Leclercq, C.; Renaut, J.; Cunha, J.; Eiras-Dias, J.; Cordeiro, C.; Matos, A.R.; Silva, M.S.; et al. An Apoplastic Fluid Extraction Method for the Characterization of Grapevine Leaves Proteome and Metabolome from a Single Sample. Physiol. Plant 2021, 171, 343-357. [CrossRef]

15. Savoi, S.; Wong, D.C.J.; Arapitsas, P.; Miculan, M.; Bucchetti, B.; Peterlunger, E.; Fait, A.; Mattivi, F.; Castellarin, S.D. Transcriptome and Metabolite Profiling Reveals That Prolonged Drought Modulates the Phenylpropanoid and Terpenoid Pathway in White Grapes (Vitis vinifera L.). BMC Plant Biol. 2016, 16, 67. [CrossRef]

16. Savoi, S.; Wong, D.C.J.; Degu, A.; Herrera, J.C.; Bucchetti, B.; Peterlunger, E.; Fait, A.; Mattivi, F.; Castellarin, S.D. Multi-Omics and Integrated Network Analyses Reveal New Insights into the Systems Relationships between Metabolites, Structural Genes, and Transcriptional Regulators in Developing Grape Berries (Vitis vinifera L.) Exposed to Water Deficit. Front. Plant Sci. 2017, 8. [CrossRef] [PubMed]

17. Spicer, R.A.; Steinbeck, C. A Lost Opportunity for Science: Journals Promote Data Sharing in Metabolomics but Do Not Enforce It. Metabolomics 2017, 14, 16. [CrossRef] [PubMed]

18. Sumner, L.W.; Amberg, A.; Barrett, D.; Beale, M.H.; Beger, R.; Daykin, C.A.; Fan, T.W.-M.; Fiehn, O.; Goodacre, R.; Griffin, J.L.; et al. Proposed Minimum Reporting Standards for Chemical Analysis. Metabolomics 2007, 3, 211-221. [CrossRef]

19. Ulaszewska, M.M.; Weinert, C.H.; Trimigno, A.; Portmann, R.; Lacueva, C.A.; Badertscher, R.; Brennan, L.; Brunius, C.; Bub, A.; Capozzi, F.; et al. Nutrimetabolomics: An Integrative Action for Metabolomic Analyses in Human Nutritional Studies. Mol. Nutr. Food Res. 2019, 63, 1800384. [CrossRef]

20. Alseekh, S.; Aharoni, A.; Brotman, Y.; Contrepois, K.; D'Auria, J.; Ewald, J.; Ewald, J.C.; Fraser, P.D.; Giavalisco, P.; Hall, R.D.; et al. Mass Spectrometry-Based Metabolomics: A Guide for Annotation, Quantification and Best Reporting Practices. Nat. Methods 2021, 18, 747-756. [CrossRef]

21. Haug, K.; Salek, R.M.; Conesa, P.; Hastings, J.; de Matos, P.; Rijnbeek, M.; Mahendraker, T.; Williams, M.; Neumann, S.; RoccaSerra, P.; et al. MetaboLights-An Open-Access General-Purpose Repository for Metabolomics Studies and Associated Meta-Data. Nucleic Acids Res. 2013, 41, D781-D786. [CrossRef]

22. Haug, K.; Cochrane, K.; Nainala, V.C.; Williams, M.; Chang, J.; Jayaseelan, K.V.; O’Donovan, C. MetaboLights: A Resource Evolving in Response to the Needs of Its Scientific Community. Nucleic Acids Res. 2020, 48, D440-D444. [CrossRef] [PubMed]

23. Harrow, J.; Drysdale, R.; Smith, A.; Repo, S.; Lanfear, J.; Blomberg, N. ELIXIR: Providing a Sustainable Infrastructure for Life Science Data at European Scale. Bioinformatics 2021, 37, 2506-2511. [CrossRef] [PubMed]

24. Sud, M.; Fahy, E.; Cotter, D.; Azam, K.; Vadivelu, I.; Burant, C.; Edison, A.; Fiehn, O.; Higashi, R.; Nair, K.S.; et al. Metabolomics Workbench: An International Repository for Metabolomics Data and Metadata, Metabolite Standards, Protocols, Tutorials and Training, and Analysis Tools. Nucleic Acids Res. 2016, 44, D463-D470. [CrossRef] [PubMed]

25. Wilkinson, M.D.; Dumontier, M.; Aalbersberg, I.J.; Appleton, G.; Axton, M.; Baak, A.; Blomberg, N.; Boiten, J.-W.; da Silva Santos, L.B.; Bourne, P.E.; et al. The FAIR Guiding Principles for Scientific Data Management and Stewardship. Sci. Data 2016, $3,160018$. [CrossRef] [PubMed]

26. Papoutsoglou, E.A.; Faria, D.; Arend, D.; Arnaud, E.; Athanasiadis, I.N.; Chaves, I.; Coppens, F.; Cornut, G.; Costa, B.V.; Ćwiek-Kupczyńska, H.; et al. Enabling Reusability of Plant Phenomic Datasets with MIAPPE 1.1. New Phytol. 2020, 227, 260-273. [CrossRef] [PubMed]

27. Baggiolini, M. Les Stades Repères Dans Le Développement Annuel de La Vigne et Leur Utilisation Pratique. Rev. Romande D'agriculture Et D'arboriculture 1952, 8, 4-6.

28. Coombe, B.G. Growth Stages of the Grapevine: Adoption of a System for Identifying Grapevine Growth Stages. Aust. J. Grape Wine Res. 1995, 1, 104-110. [CrossRef]

29. Lorenz, D.H.; Eichhorn, K.W.; Bleiholder, H.; Klose, R.; Meier, U.; Weber, E. Growth Stages of the Grapevine: Phenological Growth Stages of the Grapevine (Vitis vinifera L. Ssp. Vinifera)—Codes and Descriptions According to the Extended BBCH Scalet. Aust. J. Grape Wine Res. 1995, 1, 100-103. [CrossRef]

30. Destrac-Irvine, A.; Barbeau, G.; de Resseguier, L.; Dufourcq, T.; Dumas, V.; de Cortazar-Atauri, I.G.; Ojeda, H.; Saurin, N.; van Leeuwen, C.; Duchêne, É. Measuring the Phenology to More Effectively Manage the Vineyard. IVES Tech. Rev. Vine Wine 2019. [CrossRef] 
31. Schymanski, E.L.; Jeon, J.; Gulde, R.; Fenner, K.; Ruff, M.; Singer, H.P.; Hollender, J. Identifying Small Molecules via High Resolution Mass Spectrometry: Communicating Confidence. Environ. Sci. Technol. 2014, 48, 2097-2098. [CrossRef] [PubMed]

32. Ciubotaru, R.M.; Franceschi, P.; Zulini, L.; Stefanini, M.; Škrab, D.; Rossarolla, M.D.; Robatscher, P.; Oberhuber, M.; Vrhovsek, U.; Chitarrini, G. Mono-Locus and Pyramided Resistant Grapevine Cultivars Reveal Early Putative Biomarkers Upon Artificial Inoculation With Plasmopara Viticola. Front. Plant Sci. 2021, 12, 1297. [CrossRef]

33. Moro, L.; Da Ros, A.; da Mota, R.V.; Purgatto, E.; Mattivi, F.; Arapitsas, P. LC-MS Untargeted Approach Showed That Methyl Jasmonate Application on Vitis labrusca L. Grapes Increases Phenolics at Subtropical Brazilian Regions. Metabolomics 2020, 16, 18. [CrossRef]

34. Sun, L.; Zhu, B.; Zhang, X.; Wang, H.; Yan, A.; Zhang, G.; Wang, X.; Xu, H. The Accumulation Profiles of Terpene Metabolites in Three Muscat Table Grape Cultivars through HS-SPME-GCMS. Sci. Data 2020, 7, 5. [CrossRef] [PubMed]

35. Stefanini, I.; Carlin, S.; Tocci, N.; Albanese, D.; Donati, C.; Franceschi, P.; Paris, M.; Zenato, A.; Tempesta, S.; Bronzato, A.; et al. Core Microbiota and Metabolome of Vitis vinifera L. Cv. Corvina Grapes and Musts. Front. Microbiol. 2017, 8, 457. [CrossRef]

36. Picone, G.; Savorani, F.; Trimigno, A.; Mezzetti, B.; Capozzi, F.; Engelsen, S.B. Metabolic Changes of Genetically Engineered Grapes (Vitis vinifera L.) Studied by 1H-NMR, Metabolite Heatmaps and IPLS. Metabolomics 2016, 12, 150. [CrossRef]

37. Narduzzi, L.; Stanstrup, J.; Mattivi, F. Comparing Wild American Grapes with Vitis Vinifera: A Metabolomics Study of Grape Composition. J. Agric. Food Chem. 2015, 63, 6823-6834. [CrossRef] [PubMed]

38. Wehrens, R.; Carvalho, E.; Masuero, D.; Juan, A.D.; Martens, S. High-Throughput Carotenoid Profiling Using Multivariate Curve Resolution. Anal. Bioanal. Chem. 2013, 405, 5075-5086. [CrossRef]

39. Dal Santo, S.; Tornielli, G.B.; Zenoni, S.; Fasoli, M.; Farina, L.; Anesi, A.; Guzzo, F.; Delledonne, M.; Pezzotti, M. The Plasticity of the Grapevine Berry Transcriptome. Genome Biol. 2013, 14, r54. [CrossRef] [PubMed]

40. Mascellani, A.; Hoca, G.; Babisz, M.; Krska, P.; Kloucek, P.; Havlik, J. 1H NMR Chemometric Models for Classification of Czech Wine Type and Variety. Food Chem. 2021, 339, 127852. [CrossRef] [PubMed]

41. Arapitsas, P.; Ugliano, M.; Marangon, M.; Piombino, P.; Rolle, L.; Gerbi, V.; Versari, A.; Mattivi, F. Use of Untargeted Liquid Chromatography-Mass Spectrometry Metabolome To Discriminate Italian Monovarietal Red Wines, Produced in Their Different Terroirs. J. Agric. Food Chem. 2020, 68, 13353-13366. [CrossRef]

42. Savoi, S.; Herrera, J.C.; Carlin, S.; Lotti, C.; Bucchetti, B.; Peterlunger, E.; Castellarin, S.D.; Mattivi, F. From Grape Berries to Wines: Drought Impacts on Key Secondary Metabolites. OENO One 2020, 54, 569-582. [CrossRef]

43. Beckner Whitener, M.E.; Stanstrup, J.; Panzeri, V.; Carlin, S.; Divol, B.; Du Toit, M.; Vrhovsek, U. Untangling the Wine Metabolome by Combining Untargeted SPME-GCxGC-TOF-MS and Sensory Analysis to Profile Sauvignon Blanc Co-Fermented with Seven Different Yeasts. Metabolomics 2016, 12, 53. [CrossRef]

44. Franceschi, P.; Mylonas, R.; Shahaf, N.; Scholz, M.; Arapitsas, P.; Masuero, D.; Weingart, G.; Carlin, S.; Vrhovsek, U.; Mattivi, F.; et al. MetaDB a Data Processing Workflow in Untargeted MS-Based Metabolomics Experiments. Front. Bioeng. Biotechnol. 2014, 2, 72. [CrossRef] [PubMed]

45. Arapitsas, P.; Speri, G.; Angeli, A.; Perenzoni, D.; Mattivi, F. The Influence of Storage on the "Chemical Age" of Red Wines. Metabolomics 2014, 10, 816-832. [CrossRef]

46. Skogerson, K.; Runnebaum, R.; Wohlgemuth, G.; de Ropp, J.; Heymann, H.; Fiehn, O. Comparison of Gas ChromatographyCoupled Time-of-Flight Mass Spectrometry and 1H Nuclear Magnetic Resonance Spectroscopy Metabolite Identification in White Wines from a Sensory Study Investigating Wine Body. J. Agric. Food Chem. 2009, 57, 6899-6907. [CrossRef]

47. Ciubotaru, R.M.; Franceschi, P.; Zulini, L.; Stefanini, M.; Škrab, D.; Rossarolla, M.D.; Robatscher, P.; Oberhuber, M.; Vrhovsek, U.; Chitarrini, G. Mono-Locus and Pyramided Resistant Grapevine Cultivars Reveal Early Putative Biomarkers upon Artificial Inoculation with Plasmopara Viticola. 2021. MetaboLights. Available online: https://www.ebi.ac.uk/metabolights/MTBLS2876 (accessed on 30 October 2021).

48. Moro, L.; Da Ros, A.; da Mota, R.V.; Purgatto, E.; Mattivi, F.; Arapitsas, P. LC-MS Untargeted Approach Showed that Methyl Jasmonate Application on Vitis labrusca L. Grapes Increases Phenolics at Subtropical Brazilian Regions. 2020. MetaboLights. Available online: https:/ / www.ebi.ac.uk/metabolights /MTBLS784 (accessed on 30 October 2021).

49. Sun, L.; Zhu, B.; Zhang, X.; Wang, H.; Yan, A.; Zhang, G.; Wang, X.; Xu, H. The Accumulation Profiles of Terpene Metabolites in Three Muscat Table Grape Cultivars through HS-SPME-GCMS. 2019. MetaboLights. Available online: https://www.ebi.ac.uk/ metabolights/MTBLS968 (accessed on 30 October 2021).

50. Savoi, S.; Wong, D.C.J.; Degu, A.; Herrera, J.C.; Bucchetti, B.; Peterlunger, E.; Fait, A.; Mattivi, F.; Castellarin, S.D. Multi-Omics and Integrated Network Analyses Reveal New Insights into the Systems Relationships between Metabolites, Structural Genes, and Transcriptional Regulators in Developing Grape Berries (Vitis vinifera L.) Exposed to Water Deficit (Phenolics; UPLC-MS/MS assay). 2019. MetaboLights. Available online: https:/ / www.ebi.ac.uk/metabolights/MTBLS898 (accessed on 30 October 2021).

51. Savoi, S.; Wong, D.C.J.; Degu, A.; Herrera, J.C.; Bucchetti, B.; Peterlunger, E.; Fait, A.; Mattivi, F.; Castellarin, S.D. Multi-Omics and Integrated Network Analyses Reveal New Insights into the Systems Relationships between Metabolites, Structural Genes, and Transcriptional Regulators in Developing Grape Berries (Vitis vinifera L.) Exposed to Water Deficit (Volatile organic compounds; SPME-GC-MS assay). 2021. MetaboLights. Available online: https://www.ebi.ac.uk/metabolights/MTBLS982 (accessed on 30 October 2021).

52. Savoi, S.; Wong, D.C.J.; Degu, A.; Herrera, J.C.; Bucchetti, B.; Peterlunger, E.; Fait, A.; Mattivi, F.; Castellarin, S.D. Multi-Omics and Integrated Network Analyses Reveal New Insights into the Systems Relationships between Metabolites, Structural Genes, 
and Transcriptional Regulators in Developing Grape Berries (Vitis vinifera L.) Exposed to Water Deficit (Carotenoids; UPLC-DAD assay). 2021. MetaboLights. Available online: https://www.ebi.ac.uk/metabolights/MTBLS984 (accessed on 30 October 2021).

53. Stefanini, I.; Carlin, S.; Tocci, N.; Albanese, D.; Donati, C.; Franceschi, P.; Paris, M.; Zenato, A.; Tempesta, S.; Bronzato, A.; et al. Core Microbiota and Metabolome of Vitis vinifera L. Cv. Corvina Grapes and Musts. 2017. MetaboLights. Available online: https://www.ebi.ac.uk/metabolights/MTBLS392 (accessed on 30 October 2021).

54. Savoi, S.; Wong, D.C.J.; Arapitsas, P.; Miculan, M.; Bucchetti, B.; Peterlunger, E.; Fait, A.; Mattivi, F.; Castellarin, S.D. Transcriptome and Metabolite Profiling Reveals that Prolonged Drought Modulates the Phenylpropanoid and Terpenoid Pathway in White Grapes (Vitis vinifera L.) (Phenolics; UPLC-MS/MS Assay). 2019. MetaboLights. Available online: https://www.ebi.ac.uk/ metabolights /MTBLS897 (accessed on 30 October 2021).

55. Savoi, S.; Wong, D.C.J.; Arapitsas, P.; Miculan, M.; Bucchetti, B.; Peterlunger, E.; Fait, A.; Mattivi, F.; Castellarin, S.D. Transcriptome and Metabolite Profiling Reveals that Prolonged Drought Modulates the Phenylpropanoid and Terpenoid Pathway in White Grapes (Vitis vinifera L.) (Volatile Organic Compounds; SPME-GC-MS Assay). 2021. MetaboLights. Available online: https: //www.ebi.ac.uk/metabolights/MTBLS892 (accessed on 30 October 2021).

56. Savoi, S.; Wong, D.C.J.; Arapitsas, P.; Miculan, M.; Bucchetti, B.; Peterlunger, E.; Fait, A.; Mattivi, F.; Castellarin, S.D. Transcriptome and Metabolite Profiling Reveals that Prolonged Drought Modulates the Phenylpropanoid and Terpenoid Pathway in White Grapes (Vitis vinifera L.) (Carotenoids; UPLC-DAD Assay). 2021. MetaboLights. Available online: https://www.ebi.ac.uk/ metabolights /MTBLS889 (accessed on 30 October 2021).

57. Picone, G.; Savorani, F.; Trimigno, A.; Mezzetti, B.; Capozzi, F.; Engelsen, S.B. Metabolic Changes of Genetically Engineered Grapes (Vitis vinifera L.) Studied by 1H-NMR, Metabolite Heatmaps and IPLS. 2016. MetaboLights. Available online: https: / / www.ebi.ac.uk/metabolights/MTBLS346 (accessed on 30 October 2021).

58. Narduzzi, L.; Stanstrup, J.; Mattivi, F. The Measurement of Spatial Distribution of Grape Metabolites in Berry Tissues Using LC-MS. 2015. MetaboLights. Available online: https://www.ebi.ac.uk/metabolights/MTBLS209 (accessed on 30 October 2021).

59. Wehrens, R.; Carvalho, E.; Masuero, D.; de Juan, A.; Martens, S. High-Throughput Carotenoid Profiling Using Multivariate Curve Resolution (Stability of Isoprenoids in Grape Extract). 2014. MetaboLights. Available online: https://www.ebi.ac.uk/ metabolights/MTBLS85 (accessed on 30 October 2021).

60. Dal Santo, S.; Tornielli, G.B.; Zenoni, S.; Fasoli, M.; Farina, L.; Anesi, A.; Guzzo, F.; Delledonne, M.; Pezzotti, M. The Plasticity of the Grapevine Berry Transcriptome. 2013. MetaboLights. Available online: https://www.ebi.ac.uk/metabolights/MTBLS39 (accessed on 30 October 2021).

61. Mascellani, A.; Hoca, G.; Babisz, M.; Krska, P.; Kloucek, P.; Havlik, J. 1H NMR Chemometric Models for Classification of Czech Wine Type and Variety. 2020. MetaboLights. Available online: https://www.ebi.ac.uk/metabolights/MTBLS1677 (accessed on 30 October 2021).

62. Arapitsas, P.; Ugliano, M.; Marangon, M.; Piombino, P.; Rolle, L.; Gerbi, V.; Versari, A.; Mattivi, F. Use of Untargeted Liquid Chromatography-Mass Spectrometry Metabolome To Discriminate Italian Monovarietal Red Wines, Produced in Their Different Terroirs. 2020. MetaboLights. Available online: https://www.ebi.ac.uk/metabolights/MTBLS1443 (accessed on 30 October 2021).

63. Savoi, S.; Herrera, J.C.; Carlin, S.; Lotti, C.; Bucchetti, B.; Peterlunger, E.; Castellarin, S.D.; Mattivi, F. From Grape Berries to Wines: Drought Impacts on Key Secondary Metabolites. 2021. MetaboLights. Available online: https://www.ebi.ac.uk/metabolights/ MTBLS2330 (accessed on 30 October 2021).

64. Beckner Whitener, M.E.; Stanstrup, J.; Panzeri, V.; Carlin, S.; Divol, B.; Du Toit, M.; Vrhovsek, U. Untangling the Wine Metabolome by Combining Untargeted SPME-GCxGC-TOF-MS and Sensory Analysis to Profile Sauvignon Blanc Co-Fermented with Seven Different Yeasts. 2015. MetaboLights. Available online: https://www.ebi.ac.uk/metabolights/MTBLS212 (accessed on 30 October 2021).

65. Franceschi, P.; Mylonas, R.; Shahaf, N.; Scholz, M.; Arapitsas, P.; Masuero, D.; Weingart, G.; Carlin, S.; Vrhovsek, U.; Mattivi, F.; et al. MetaDB a Data Processing Workflow in Untargeted MS-Based Metabolomics Experiments. 2014. MetaboLights. Available online: https:/ / www.ebi.ac.uk/metabolights/MTBLS137 (accessed on 30 October 2021).

66. Arapitsas, P.; Speri, G.; Angeli, A.; Perenzoni, D.; Mattivi, F. The Influence of Storage on the "Chemical Age" of Red Wines. 2014. MetaboLights. Available online: https://www.ebi.ac.uk/metabolights/MTBLS55 (accessed on 30 October 2021).

67. Skogerson, K.; Runnebaum, R.; Wohlgemuth, G.; de Ropp, J.; Heymann, H.; Fiehn, O. White Wine Study. 2013. MetaboLights. Available online: https: / / www.metabolomicsworkbench.org/data/DRCCMetadata.php?Mode=Project\&ProjectID=PR000005 (accessed on 30 October 2021).

68. Powers, S.M.; Hampton, S.E. Open Science, Reproducibility, and Transparency in Ecology. Ecol. Appl. 2019, 29, e01822. [CrossRef] [PubMed] 